\title{
Radiographic Evaluation of Heart Using VHS method in Rajapalayam Dog- Indigenous Breed of Tamil Nadu
}

\author{
S.Bhargavi ${ }^{1}$, T.A. Kannan ${ }^{1 *}$, Geetha Ramesh ${ }^{1}$, D. Sumathi ${ }^{2}$ and A. Arun Prasad ${ }^{3}$ \\ ${ }^{1}$ Department of Veterinary Anatomy, ${ }^{2}$ Department of Veterinary Clinical Medicine, \\ ${ }^{3}$ Department of Veterinary Surgery and Radiology, Madras Veterinary College, \\ Chennai-07, Tamil Nadu, India \\ *Corresponding author
}

\section{A B S T R A C T}

\section{Keywords}

Vertebral heart score, Indigenous dog, Rajapalayam, Heart, Radiography

Article Info

Accepted:

10 January 2019

Available Online:

10 February 2019

\begin{abstract}
Now-a-days, VHS measurements were recorded to assess the cardiac silhouette and size in various exotic dog breeds in the world. However, there is little or no work on cardiac anatomy in Indigenous dog breeds of Tamil Nadu such as Rajapalayam. Rajapalayam dog which is a unique medium-sized, sight hound breed of Rajapalayam region in the Virudhunagar district of southern Tamil Nadu. In the present study, Vertebral heart score (VHS) was measured by thoracic radiographs of left and right lateral recumbency. A total of 12 dogs (young -6 and adult- 6 ) were used for the study. The mean VHS on right lateral radiographs was found to be 9.07 and 9.08 vertebrae in young and adult dogs respectively. Whereas, the mean VHS on left lateral radiographs was found to be 9.10 and 9.08 vertebrae in young and adult dogs respectively. No significant differences could be found between the VHS of both young and adult Rajapalayam dogs. As breed wise variations are observed in the VHS measurements, this study documents the VHS measurements of Rajapalalaym dog.
\end{abstract}

\section{Introduction}

Heart, the largest organ in the middle mediastinum, is located between the walls of the mediastinal pleura which is in turn was partly divided transversely into the bloodreceiving chambers, the atria and the pumping chambers, the ventricles. The musculature and conducting system of the heart was spoken collectively as the myocardium (Evans, 1993).

The heart was located slightly obliquely right to left, in the ventral two-thirds of thorax, extended approximately from third to sixth intercostal spaces, slightly more than half to the left of the midline (Anderson and Anderson, 1994 and Adams, 2004).

Radiographs of thoracic cavity are useful as another diagnostic modality to detect heart diseases and has the potential to provide information equivalent to other cardiac diagnostic modalities. The vertebral heart score (VHS) involves measuring long axis and short axis of heart from lateral radiographs of thorax. Knowledge of interbreed variation in thoracic conformation 
and selection of proper reference value of VHS in diagnosis of heart enlargement in dogs (Gugjoo et al., 2013).

India has ample canine genetic resource, with several indigenous canine breeds such as Rajapalayam, Chippiparai, Kombai, Kanni, Mudhol hound, Bully Kutta and Gaddi were well known. Of which, Rajapalayam dog, a unique medium-sized, sight hound breed. It was described as the companion of royalty and aristocracy in southern India, particularly in the town of Rajapalayam in Virudhunagar district and also known as Paleiyakaran and Poligar hound (Srinivasan, 2011).

Hence, the present study was designed to develop the basic data on cardiac size in Rajapalayam breed using Digital Radiography.

\section{Materials and Methods}

\section{Study area}

The study was carried out on 12 clinically healthy Rajapalayam breeds of dogs brought to Madras Veterinary College teaching hospital with the consent of the owner. The dogs were grouped into two groups based on age as young / puppy (from 1 to 6 months) and adult (6 months and above). Each group consisted of six animals.

\section{Screening}

Animals were screened initially to ascertain that they were clinically healthy by physical examination and clinical examination. The history and signalments were noted. A thorough physical examination was carried out and vital parameters like respiration rate (per minute), heart rate (beats per minute), rectal temperature $\left({ }^{\circ} \mathrm{C}\right)$ were recorded. Dogs with vital parameters within the established reference range were considered to be clinically healthy and subjected for further evaluation.

\section{Thoracic radiography}

The radiographic examination included left and right lateral thoracic radiographs from non-sedated animals.

While radiography, care was taken to avoid movement of the animal so that the shape and size of the cardiac silhouette was not deviated from normal (Bavegems et al., 2005). Radiographic images were obtained by using Siemens $500 \mathrm{~mA}, 3$ phase, 6 pulse X-ray generator. Digital processing of the images was carried out using Computerised radiography AGFA 30-X using the standard exposure technique based on the chest depth of the animal.

\section{Measurements}

Measurement of the Vertebral Heart Score (VHS) was done as per Buchanan and Bucheler (1995) in right and left lateral views of thoracic radiographs in dogs.

Vertebral Heart Score $=$ Short axis dimension + Long axis dimension

It was expressed as the total units of vertebral length to the nearest 0.1 vertebra.

The long axis (LA) and short axis (SA) were measured in right-to-left and left-to-right lateral radiographs beginning from the cranial edge of $\mathrm{T} 4$.

\section{Statistical analysis}

Statistical analysis was performed using SPSS software (SPSS ${ }^{\circledR} 20.0$ for Windows). Results were expressed as a mean \pm standard error. $\mathrm{T}$ test for equality of means was used to compare the differences between young and 
adult age groups and left lateral versus right lateral VHS respectively.

\section{Results and Discussion}

Thoracic radiographs were taken from both left and right lateral recumbency in unsedated Rajapalayam dogs using Digital Radiography.

Long axis and short axis of heart were measured in both left and right thoracic radiographs in young and adult age groups.
VHS values were calculated for both left and right lateral recumbenccy in both the age groups studied. Comparison was made between the two age groups (Table 1 and Figure 1).

T-test for equality of means was performed to detect the significant difference between the two age groups studied (young and adult). No significant difference $(\mathrm{P}>0.05)$ was observed in VHS measurements in both recumbencies between the age groups.

Table.1 Mean \pm SE of Vertebral heart score in Rajapalayam dogs

\begin{tabular}{|l|c|c|c|c|}
\hline \multicolumn{2}{|c|}{ Parameters } & Young & Adult & t value \\
\hline \multirow{2}{*}{ Left Lateral } & LA & $5.05 \pm 0.60$ & $5.05 \pm 0.02$ & $0.00^{\text {NS }}$ \\
\cline { 2 - 5 } & SA & $4.05 \pm 0.04$ & $4.03 \pm 0.04$ & $0.28^{\text {NS }}$ \\
\cline { 2 - 5 } Right Lateral & VHS & $9.1 \pm 0.07$ & $9.08 \pm 0.05$ & $0.19^{\text {NS }}$ \\
\cline { 2 - 5 } & LA & $5.02 \pm 0.06$ & $5.03 \pm 0.10$ & $0.15^{\text {NS }}$ \\
\cline { 2 - 5 } & SA & $4.05 \pm 0.01$ & $4.05 \pm 0.02$ & $0.00^{\text {NS }}$ \\
\cline { 2 - 5 } & VHS & $9.07 \pm 0.13$ & $9.08 \pm 0.10$ & $0.10^{\text {NS }}$ \\
\hline
\end{tabular}

NS - No significant difference between young and adult age groups $(\mathrm{P}>0.05)$

Figure.1 Graphical representation of Mean \pm SE of Vertebral Heart Score in Rajapalayam dogs $\mathrm{X}$ axis denotes Parameters, $\mathrm{Y}$ axis denotes VHS value

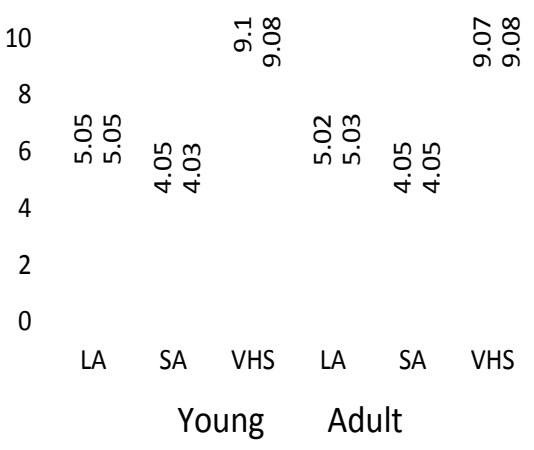


Figure.2 Right lateral Radiographs of Rajapalayam dog showing normal cardiac anatomy

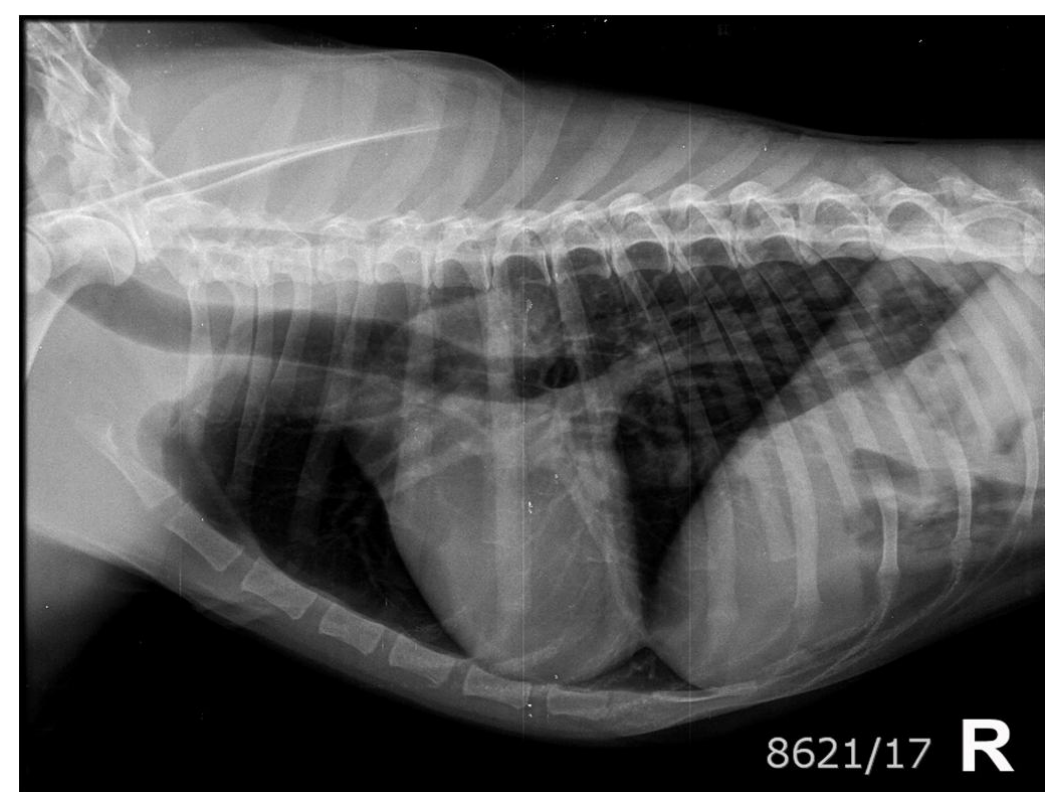

Figure.3 Left lateral Radiographs of Rajapalayam dog showing VHS measurements $\mathrm{L}$ - Long axis of heart $\quad \mathrm{S}$ - Short axis of heart

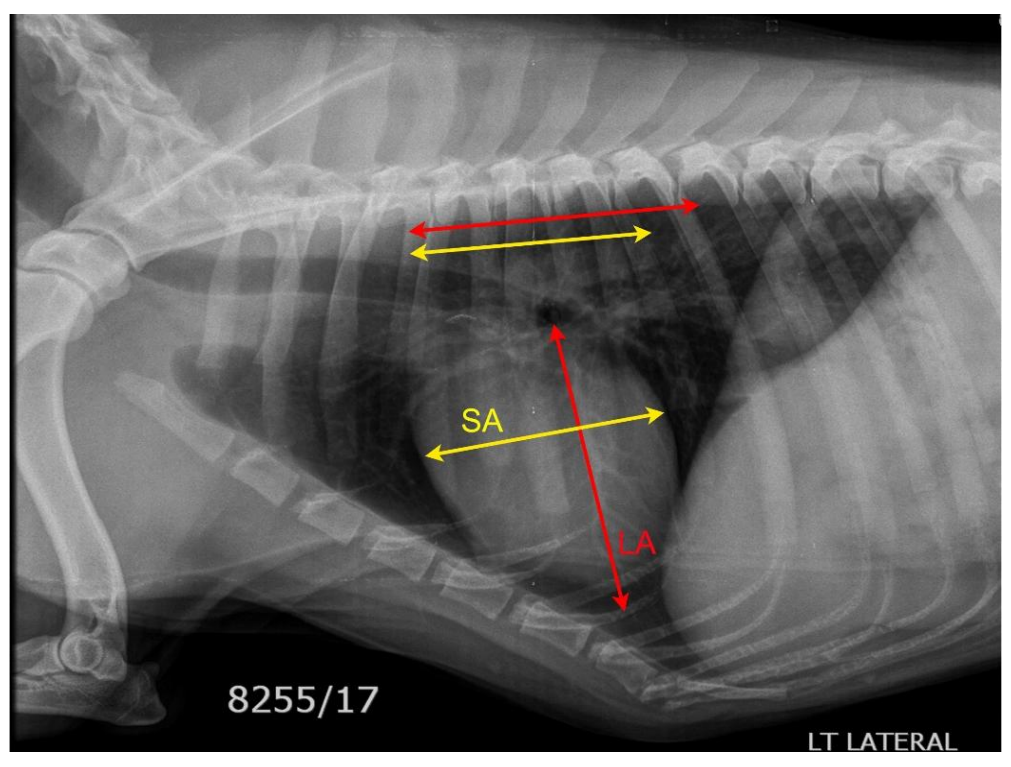

\section{References}

Adams, D.R., 2004. Canine Anatomy, A Systemic Study. $4^{\text {th }}$ edition. WilleyBlackwell.

Anderson, W. D. and Anderson, B.G., 1994.
Atlas of Canine Anatomy. $1^{\text {st }}$ Edition. Hagerstown, Maryland, U.S.A.: Lea \& Febiger.

Bavegems, V., Van Caelenberg, A. Duchateau, L., Sys, S.U., Van Bree, H. et al., 2005, Vertebral heart size ranges 
specific for whippets, Veterinary Radiology \& Ultrasound 46, 5, 400403.

Bodh, D., Hoque, M., Saxena, A.C., Gugjoo, M.B,, Bist, D. et al., 2016, Vertebral scale system to measure heart size in thoracic radiographs of Indian Spitz, Labrador retriever and Mongrel dogs, Veterinary World 9, 371-376.

Buchanan, J.W. and Bucheler, H., 1995, Vertebral scale system to measure canine heart in radiographs, Journal of the American Veterinary Medical Association 206, 194-199.

Done, S.H., Goody, P.C., Evans, S.A. and Stickland, N.C., 2009, Colour Atlas of Veterinary Anatomy. Volume 3. The Dog and Cat. $2^{\text {nd }}$ edition. Elsevier Ltd.

Evans, H.E., 1993, Miller's anatomy of the dog, 3rd edition. WB Saunders, Philadelphia.

Ghadiri, A., Avizeh, R. and Fazili.G., 2010, Vertebral scale of common large breeds of dogs in Iran, Iranian Journal of Veterinary Medicine 2, 107-111.

Gugjoo, M.B., Hoque, M., Saxena, A.C., Zama, M.M.S. and Amarpal., 2013, Vertebral scale system to measure heart size in dogs in thoracic radiographs,
Advances in Animal and Veterinary Sciences 1, 1-4.

Lamb, C.R., Wilkeley, H., Boswood, A. and Pfeiffer, D.U., 2001, Use of breedspecific ranges for the vertebral heart scale as an aid to the radiographic diagnosis of cardiac disease in dogs, Veterinary Record 148, 707-711.

Marin, L.M., Brown, J. and McBrien, C., 2007, Vertebral heart size in retired racing Greyhounds, Veterinary Radiology \& Ultrasound 48, 332-334.

Sleeper, M.M. and Buchanan, J.W., 2001, Vertebral scale system to measure heart size in growing puppies, Journal of American Veterinary Medical Association 219, 57-59.

Spasojevic-Kosic, Lj., Krstic, N. and Trailovic, R.D., 2007, Comparison of three methods of measuring vertebral heart size in German Shepherd Dogs, Acta Vet (Beogr) 57, 133-141.

Srinivasan, S.R., 2011, Present status of dog genetic resources of Tamil Nadu. In: Workshop Manual on Conservation of Animal Genetic Resources of Tamil Nadu, organized by TANUVAS on 2324, June, 2011 at Chennai, Tamil Nadu.

\section{How to cite this article:}

Bhargavi, S., T.A. Kannan, Geetha Ramesh, D. Sumathi and Arun Prasad, A. 2019. Radiographic Evaluation of Heart Using VHS method in Rajapalayam Dog- Indigenous Breed of Tamil Nadu. Int.J.Curr.Microbiol.App.Sci. 8(02): 1216-1220. doi: https://doi.org/10.20546/ijcmas.2019.802.141 